\title{
Modeling and development of radio frequency planar interdigital electrode sensors
}

\author{
Muhammad Farhan Affendi bin Yunos, Anis Nurashikin Nordin, Anwar Zainuddin, Sheroz Khan \\ Department of Electrical and Computer Engineering, Kuliyyah of Engineering, \\ International Islamic University Malaysia, Malaysia
}

\begin{tabular}{l} 
Article Info \\
\hline Article history: \\
Received Feb, 2019 \\
Revised May 20, 2019 \\
Accepted Jun 11, 2019 \\
\hline
\end{tabular}

Keywords:

Capacitive sensors CST microwave studio Frequency response Interdigital electrode Permittivity sensors Planar interdigital sensor Radio frequency

\begin{abstract}
The interdigital sensor has been implemented in various field of applications such as microwave device, chemical sensor and biological sensor. This work describes the design and fabrication of an interdigital sensor (IDS) design that has the potential of estimating blood glucose levels using capacitive measurements. The IDS was first designed using theoretical equations and later was optimized by using CST Microwave Studioß. The electrode widths of the sensor were varied from $0.5 \mathrm{~mm}$ to $0.7 \mathrm{~mm}$ and the $S_{11}$ reflection characteristics were simulated. Upon completion of simulations, the sensor was fabricated using copper clad FR4 boards. The fabricated sensors were measured using a vector network analyzer (VNA) and produced resonance frequencies of 2.02, 2.11 and $2.14 \mathrm{GHz}$. The highest Q obtained was 11.72 from the $2.11 \mathrm{GHz}$ sensor.
\end{abstract}

Copyright () 2019 Institute of Advanced Engineering and Science. All rights reserved.

\section{Corresponding Author:}

Anis Nurashikin binti Nordin,

Department of Electrical and Computer Engineering,

Kulliyah of Engineering,

International Islamic University Malaysia,

Jalan Gombak, 53100, Selangor, Malaysia.

Email: anisnn@iium.edu.my

\section{INTRODUCTION}

The periodic interdigital structure is a very common design amongst electronics devices especially for sensing. Recent advances in fabrication technology for microelectromechanical systems (MEMS) have promoted the usage of the interdigital structure in different fields such as non-destructive testing, telecommunications, piezoacoustics and biosensors. The term 'interdigital' refer to the fingerlike or digitlike periodic arrangement of the planar electrodes that is used to form capacitors on a specific substrate [1]. The fingerlike structure allows multiple capacitors to be placed in parallel, which causes the capacitive values to be added together. This multiplicative effect increases the sensing density of the sensor, where a high capacitance value can be achieved in a small area. Depending on the substrate material, the interdigital capacitive sensors can be used either as electric field sensors or acoustic wave sensors. Acoustic wave sensors require the interdigital sensors to be placed on top of piezoelectric material so that the electrodes acts as a transducer and converts input electrical signal into mechanical acoustic waves [2]. The length of the periodic spacing in the interdigital pattern determines the frequency of the acoustic waves and it can be used for a wide range of applications such as sonar, surface acoustic wave sensors, acoustic-plate mode sensor, acoustic microscopes and surface acoustic wave signal processors [3].

The more traditional usage of interdigital sensors (IDS) via electric field sensing is as capacitive sensors. The interdigital electrodes generate fringing electric fields which penetrate into the material and can be used to measure changes in various physical, chemical or structural properties of the material. The usage 
of the interdigital structure has many inherent advantages namely single-port access, control of signal strength, capability of multiple detection (conductivity, permeability, permittivity) and simplified modelling [4]. Recent research have reported interdigital capacitive sensor designs that have been used as chemical sensor [5], strain gauge, proximity testing, defect sensing, food inspection [6] and humidity sensor [7]. In the case of the humidity sensor, the interdigital capacitive sensor detects changes in permittivity in accordance to fluctuations of percentage of humidity in the surroundings. In another application, interdigital capacitive sensors have been used as a self-calibrated water level measurement sensor [8]. The water level in a chamber can be measured via impedance change in between two comb electrodes. A microcontroller was used to measure a discharge time of capacitance between two comb electrodes correlated to the water level. Interdigital designs are widely popular and have also been used for accelerometer, pressure sensor, flow rate, biosensors and capacitive touch screen designs.

This paper presents the modeling and development of a planar interdigital capacitive sensor for permittivity measurements. Section 2 explains the design theory of the interdigital sensor (IDS). The $\mathrm{S}_{11}$ frequency response and quality factor with varying width and gap was analyzed. Section 3 describes the modelling and optimization of the IDS using CST Microwave Studio in air. Section 4 explains the fabrication method of the IDS sensor and its experimental measurements. Section 5 discusses the results and concludes the paper.

\section{DESIGN AND THEORY}

A planar interdigital sensor utilizes the concept of capacitive parallel plate electrodes and transforms it into fringing electric fields. Two electrode system is used to generate a uniform electric field on top of the sensor. An IDS comprise of sets of adjacent working (positive) and counter (negative) electrodes on a planar surface. AC signal will be applied to the working electrodes; generating an electric field on top of the electrodes. Electric fields are induced between two conductors through capacitive coupling. Figure 1 shows a single pair of positive and negative planar electrodes and its generated electric field. According to its basic principles, the amount of capacitance is proportional to the area between the two electrodes and is inversely proportional to the distance between the two parallel plate conductors. Induced capacitance between the two conductors separated by an insulating medium can also be described mathematically as follows:

$$
C=\frac{\varepsilon_{0} \varepsilon_{r} A}{d}
$$

where $C$ is a capacitance, $\varepsilon_{0}$ is a permittivity of free space $\left(8.854 \times 10^{-12} \mathrm{~F} / \mathrm{m}\right), \varepsilon_{r}$ is the relative permittivity of medium (constant), $A$ is the surface area of the conductor and $d$ is the distance between two conductors. Thus, larger the surface area of plate conductors and smaller the distance between two parallel plate conductors would induce more current and electric field [9]. Multiple pairs of interdigital electrodes can be connected together to increase $C$ and form an interdigital capacitive sensor as shown in Figure 1 . The variables $l, w, d$ and $t$ indicate the length, width, gap spacing between the electrodes and thickness of the electrodes respectively.
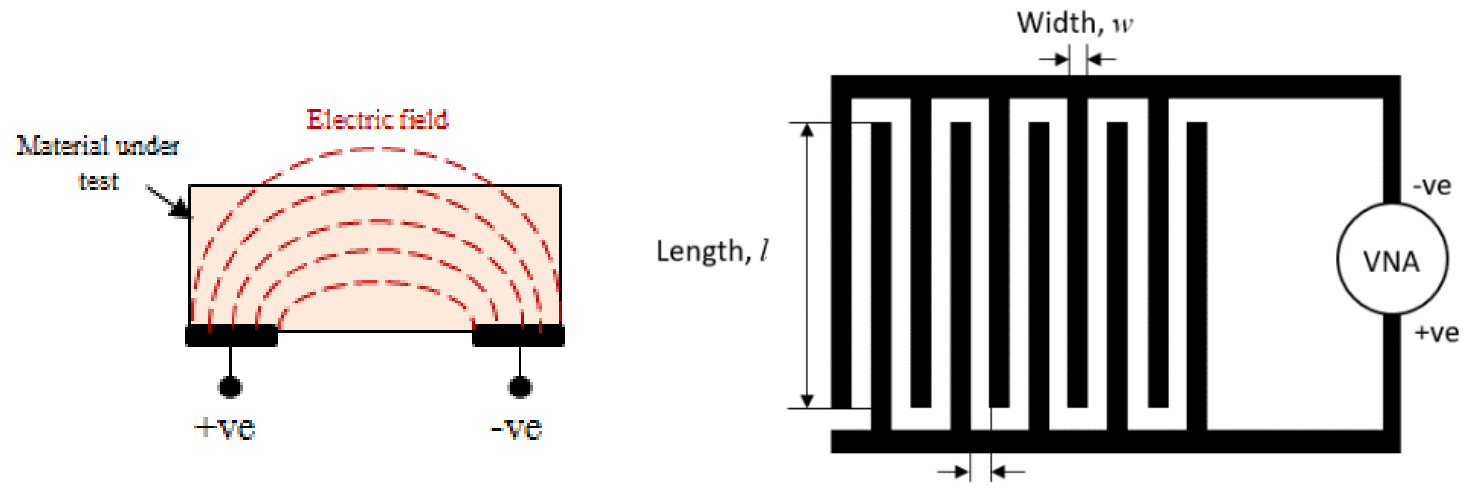

Distance, $d$

Figure 1. Left: fringing electric field in a coplanar interdigital sensor, right: configuration for coplanar interdigital sensor and its important parameters 
When placed underneath an unknown material, these capacitors generate fringing electric fields that penetrate the material under test and thus the permittivity value of the unknown material, $\varepsilon_{r}$ can be measured. To evaluate the change in capacitance we need to evaluate the change in impedance which is given in (2) as follows:

$$
Z=\frac{1}{j \omega C}=\frac{1}{j 2 \pi f C}
$$

where $\mathrm{Z}$ is the capacitive and $f$ is frequency of the maximum impedance. The frequency response of the IDS can also be evaluated in terms of its reflection coefficient $S_{11}$. When plotted at varying frequencies, the $S_{11}$ frequency response generated a resonance dip at a specific frequency, $f$. This frequency, $f$ can also be written in another form as shown below:

$$
f=\frac{1}{j 2 \pi Z} \cdot \frac{d}{\varepsilon A}=\frac{d}{j 2 \pi Z \varepsilon A}
$$

The distance, $d$ and width, $w$ were varied and the change in resonance dip, $f$ were evaluated using (3) and plotted in Figure 2(a). In this design, the ratio of $(w / d)$ is kept at $1: 1$ as a standard ratio of interdigitated electrodes configuration or $w=d$ for all designs. It can be seen that $f$ increases as $d$ increases.

Next, the theoretical evaluation of another parameter, $Q$ was done. The Quality factor or $Q$ factor of electrical components and devices is a measure of the relationship between stored energy and the rate of energy use, giving an indication of their efficiency [10]. The basic $Q$ or quality factor equation is based upon the energy losses within the inductor, circuit or other form of component. $Q$ can also be measured from the S11 frequency response using the equation below:

$$
Q=\frac{f}{\Delta f_{3 d B}}
$$

where $f$ is the frequency dip and $\Delta f_{3 d B}$ is the difference in frequencies at $3 \mathrm{~dB}$ distance to $f$. For sensing applications, it is important that the $Q$ values are high as it can produce more sensitive sensors. Figure 2(b) shows the decrease in $Q$ with increasing, $w$. This indicates that there is a tradeoff with increasing $w$ as the $Q$ starts to drop at higher frequencies. For our work, this sensor was developed for non-invasive blood glucose sensing applications where the change in glucose concentrations is detected via change in capacitance and thus its permittivity. The ideal range of frequency for blood glucose detection is $1-3 \mathrm{GHz}[11,12]$. In order to achieve higher $Q$, the electrode width was selected to be between $0.4 \mathrm{~mm}$ to $0.8 \mathrm{~mm}$. More accurate simulations were done using finite element method in the next section.

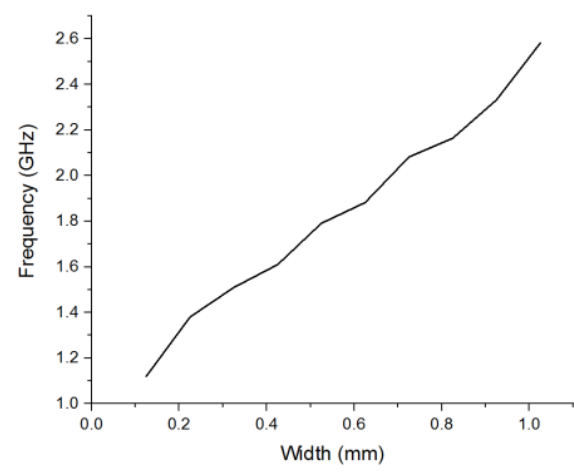

(a)

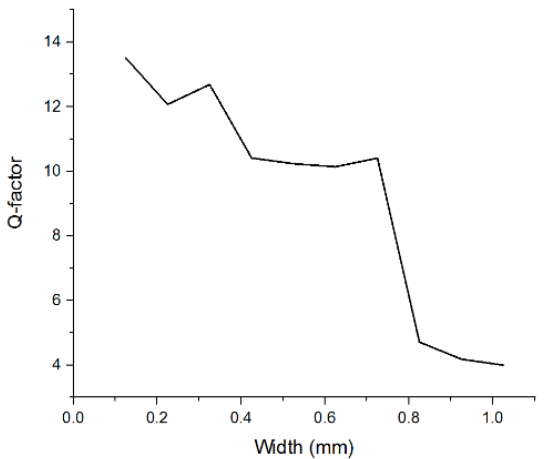

(b)

Figure 2. (a) Variations of electrode width versus frequency, (b) Variations of electrode width versus $\mathrm{Q}$-factor. Note that $w=d$ for all designs

\section{FINITE ELEMENT MODELING OF IDS}

For more accurate analysis, it is crucial to model the electric field generated by the sensor structure via finite element analysis. There is many software that can be used to analyse interdigital electrode structure, material, and design using finite element analysis [13]. In this work, CST MICROWAVE STUDIO® was

Modeling and development of radio frequency planar interdigital... (Muhammad Farhan Affendi bin Yunos) 
used to evaluate the electric field and single port S11 reflection characteristics. CST MICROWAVE STUDIO ${ }^{\circledR}$ is a specialist tool for the 3D EM simulation of high frequency components. CST MWS is frequently used to produce fast and accurate analysis of high frequency (HF) devices such as antennas, filters, couplers, planar and multi-layer structures and SI and EMC effects. Finite element simulation is very important to get the initial result using a software tool, which allows us to visualize the frequency response of the IDS sensor before fabrication. CST is used to optimize the periodic width of the IDS designs. In this work, the simulations were performed for electrode widths ranging from $0.5 \mathrm{~mm}$ to $0.7 \mathrm{~mm}$.

The interdigital sensor is formed using copper electrodes on a Flame Retardant 4 (FR4) substrate or printed circuit board commonly used for electronic circuitry. The FR4 printed circuit board has thickness of $1.57 \mathrm{~mm}$, relative permittivity, $\square_{r}$ of 4.7 and loss tangent 0.014 [14]. The important geometry parameters set in CST were as shown in Table 1.

Table 1. Sensor geometry parameter settings for CST microwave studio $®$ simulation

\begin{tabular}{cccccc}
\hline \multicolumn{2}{c}{ Layer } & $\mathrm{x}(\mathrm{mm})$ & $\mathrm{y}(\mathrm{mm})$ & $\mathrm{z}(\mathrm{mm})$ & Frequency \\
\hline \multirow{3}{*}{ Electrode } & Width $(w)$ & 0.6 & - & 0.1 & \\
& Length $(l)$ & - & 14 & 0.1 & \\
& Distance $(d)$ & 0.6 & - & 0.1 & $1-3 \mathrm{GHz}$ \\
Substrate & 50 & 50 & 1.6 & \\
Waveguide Port & - & 10 & 4.6 & \\
\hline
\end{tabular}

The 3D simulation model of the interdigital sensor in CST Microwave Studio® is shown in Figure 3(a). The input and output ports to the IDS sensor is labelled as the waveguide port. In this simulation, the length of the electrode was remained constant while $w=d$ have been varied from $0.5 \mathrm{~mm}, 0.6 \mathrm{~mm}$ and 0.7 $\mathrm{mm}$. The number of electrode pairs, $N=10$. The frequency response of the IDS was simulated between $1 \mathrm{GHz}$ to $3 \mathrm{GHz}$. The objective of the simulation is to evaluate the reflection characteristics of the three sensors and to calculate its Q-factor. CST Microwave Studio ${ }^{\circledR}$ can also evaluate the electric field distribution on the electrodes. The electric field distribution and its direction is shown in Figure 3(b).

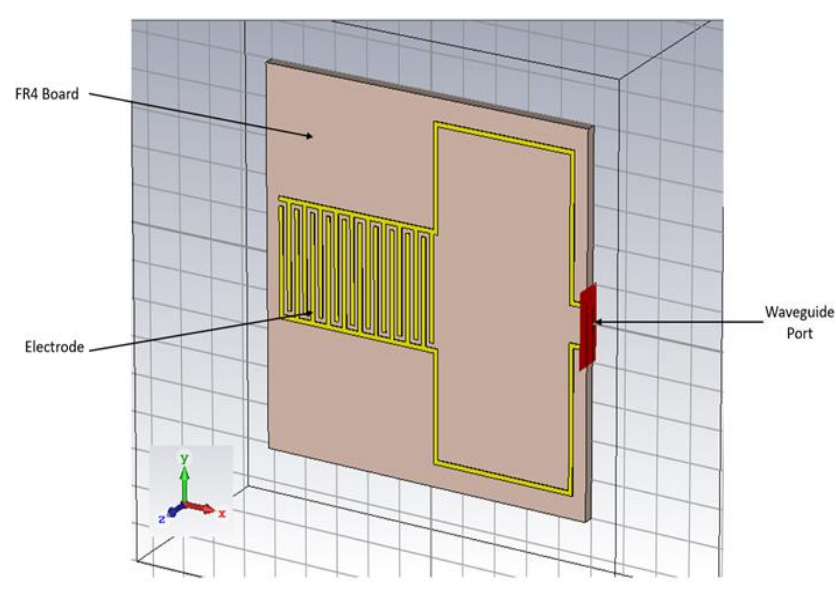

(a)

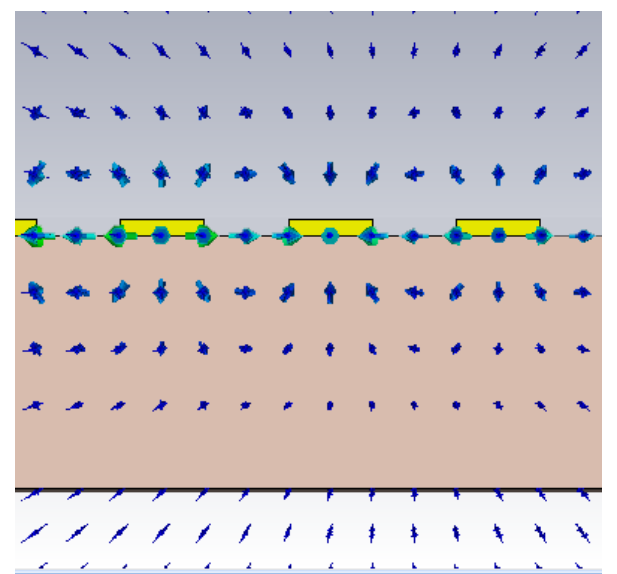

(b)

Figure 3. (a) The CST simulation sensor design of IDS, (b) The electric field distribution on the interdigital electrodes

The next important simulation for the IDS is its reflection characteristics. For this work, the S11 reflection characteristics were simulated in the frequency range of interest, between 1-3 GHz. Three different widths, $\mathrm{w}=\mathrm{d}$, were simulated namely $0.5 \mathrm{~mm}, 0.6 \mathrm{~mm}$ and $0.7 \mathrm{~mm}$. Figure 4 shows the frequency response of these three devices. The resonance dips were found to be $1.79,1.88$ and $2.06 \mathrm{GHz}$ respectively. Q-factor value for width were calculated using (4) to be 10.02, 10.13 and 10.41 respectively. It can be seen based on the simulations that the change in the electrode widths affect the frequency response of the sensor. As the width increases, the resonance frequency shifts to the right. This is correlates to (3). 


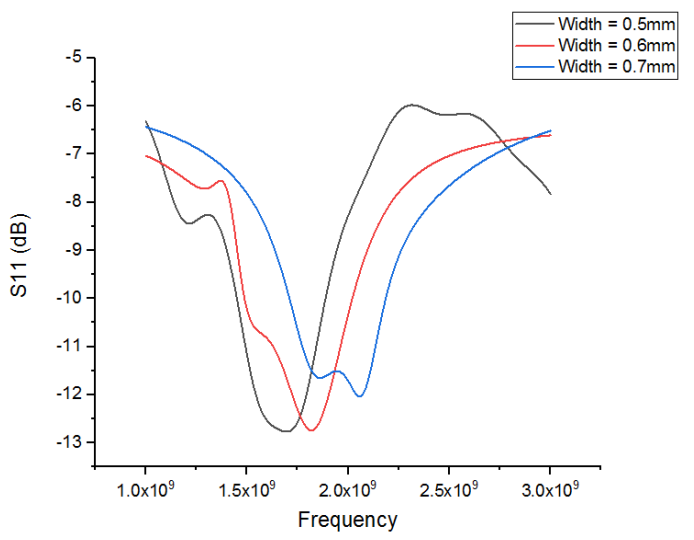

Figure 4. Simulation result with varying width of electrode

\section{EXPERIMENTAL WORK}

Once the design is finalized and its simulations are satisfactory, the next step to is to fabricate the designs on the FR4 board. This section describes both the sensor's fabrication process and its measurements conducted using a vector network analyzer.

\subsection{Fabrication steps for FR4 board}

The flame retardant 4 (FR4) board or printed circuit board consists of four main parts which is the substrate, copper layer, solder mask and silkscreen [15]. The substrate of FR4 is made of fiberglass because it helps resist breakage and provides a core strength to FR4 board. For this work we used FR4 boards that are already copper clad with a thin photoresist film on top of it. There are two options to design the masks for the boards, either using positive or negative planes. The positive planes show the actual designs being developed, while the negative plane shows the inverted design. In this work we have chosen the positive design process. The step-by-step fabrication flow is shown in Figure 5 which involves design, exposure, followed by development, etching and the final step is stripping.

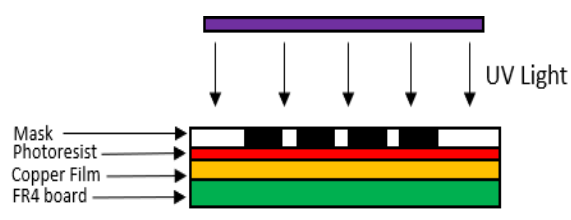

UV exposure
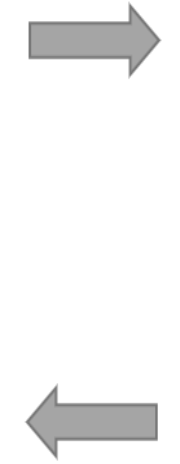

Stripping

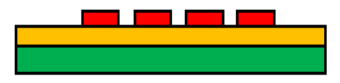

Development
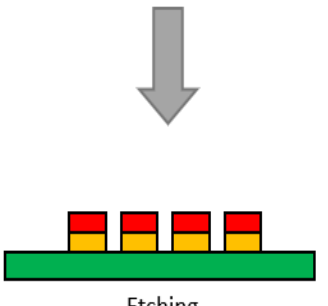

Etching

Figure 5. Fabrication flow for FR4 board

The design was first drawn in powerpoint and the printed on a transparency. Next, the printed design is attached to the positive board and is placed an UV exposure machine. The design is exposed to UV light for about 180 seconds. During this process, the UV light causes the exposed areas to be more soluble. After the exposure, the board is immersed into the developing solution, Sodium Hydroxide for about 2 to 3 minutes. The developer solution dissolves the exposed photoresist, while the unexposed photoresist is polymerized. This creates a pattern on the board. Next, the board was rubbed and cleaned with clean water. The following step is to remove the unwanted copper. This done by etching the pattern using Ferric Chloride

Modeling and development of radio frequency planar interdigital... (Muhammad Farhan Affendi bin Yunos) 
solution in the etching machine. After the etching, we can see only the traces of the design and the excess copper is completely removed. If the excess copper is not fully removed, it can be placed again in the Etching Machine. Once the etching process is done, the photoresist layer was removed through stripping process. The photoresist film is removed from the board by rubbing it gently until it is completely removed. Finally the board is rinsed with clean water and left for 5 minutes to dry. Next, the FR4 board is tested with multimeter to make sure there is no short circuit or open circuit.

The fabricated interdigital sensor is shown in Figure 6. It can be seen that the interdigital electrodes are fabricated well and there are no shorts occurring between the electrodes. For measurements, a female SMA connector is attached to the sensor.

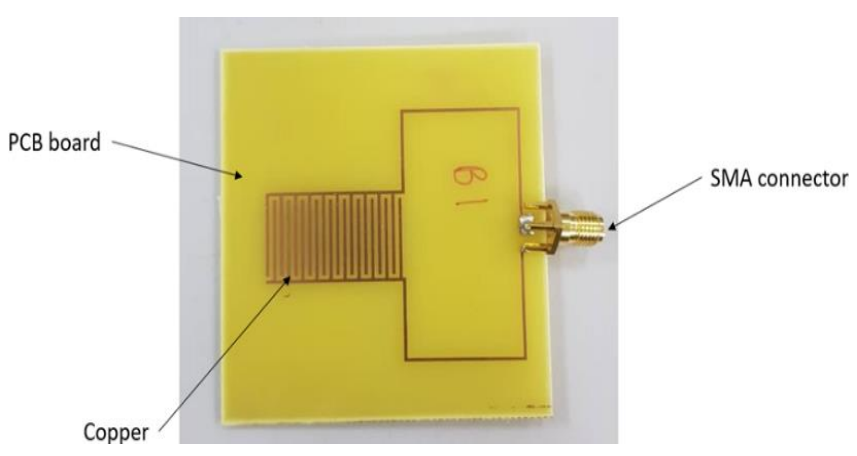

Figure. 6. The fabricated IDS sensor

\subsection{Experimental Measurements}

To validate the sensors' simulation performance the $S_{11}$ frequency response was measured experimentally with varying sensor width. Evaluations of interdigital sensor's frequency response were made using Agilent E5062A, 300kHz-3 GHz Vector Network Analyzer (VNA). The VNA is a measurement instrument that can characterize the microwave parameters, mainly the scattering parameters. The sensor was connected to female SMA connector and then to the VNA as shown in Figure 7. The VNA is used to generate RF AC signal across the sensor and measure the feedback. The sensor directly attached to VNA without using the probe to reduce the noise to get smooth signal generate by VNA.

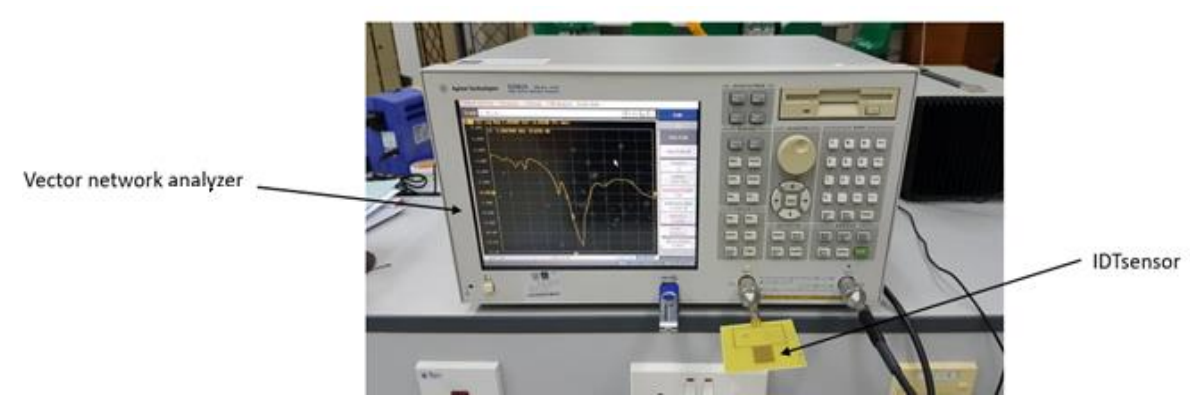

Figure 7. The measurement setup of the interdigital sensor with the Agilent E5602A vector network analyzer

Before using the VNA to measure the frequency response in this sensor, few calibrations need to be performed. The VNA have been calibrated using an Open, Short and Load (OSL) calibration with an SMA calibration kit. The calibration was done by doing a measurement of an OSL termination at the point where the sensor will be measured which is directly at the port. The calibration and measurement frequencies of the VNA was set between $1 \mathrm{GHz}$ to $3 \mathrm{GHz}$. The VNA software uses the calibration data to discard the losses at the port during measurements.

Once the calibration was completed, experimental measurements for the three sensors in the same frequency range were made. S11 reflection characteristics versus frequency were measured and plotted as shown in Figure 8. Smoothing function on the VNA was used to produce better results. The three sensor 
devices showed the same trend as the simulations where the sensor with the smallest width, 0.5 mm produces the lowest resonance dip of $2.02 \mathrm{GHz}$, followed by 2.11 for $w=0.6 \mathrm{~mm}$ and $2.14 \mathrm{GHz}$ for $w=0.7 \mathrm{~mm}$. The measured Q factors of the sensors were also calculated and found to be 10.63, 11.72 and 5.15 respectively.

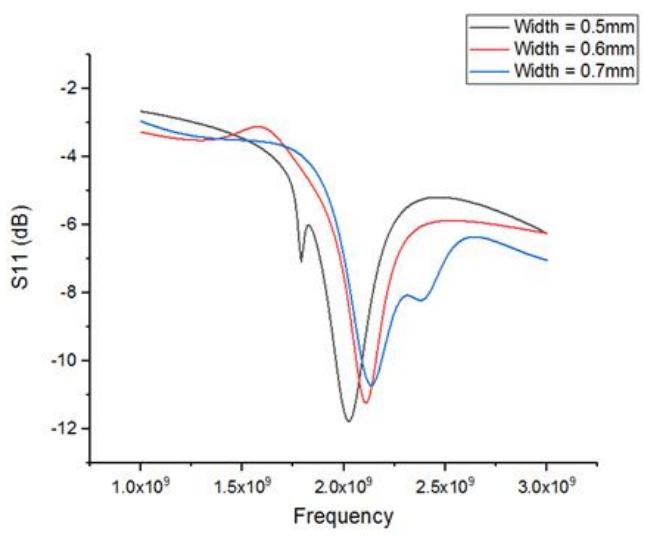

Figure 8. Measurement result with varying width of electrode

Figure 9 shows comparison plots of the simulation and measurement S11 characteristics for all three devices. While the trend of increasing resonance frequencies is shown with increased widths, there is a large difference between the simulated and measurement resonance frequencies. The difference between simulation and measurements are attributed to inaccurate modelling of the material properties of the FR4 board and the surroundings. The range of losses from -2 to $-12 \mathrm{~dB}$ is still similar for both simulation and experimental measurements.

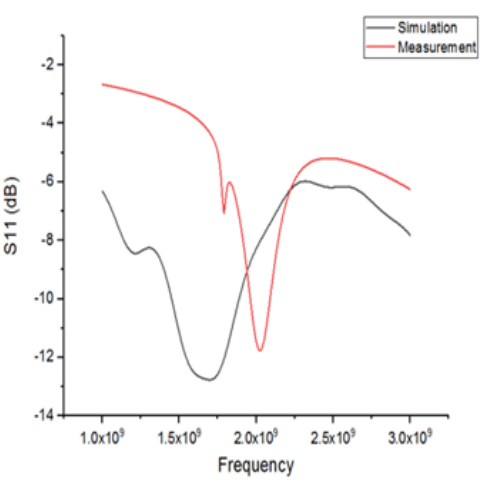

(a)

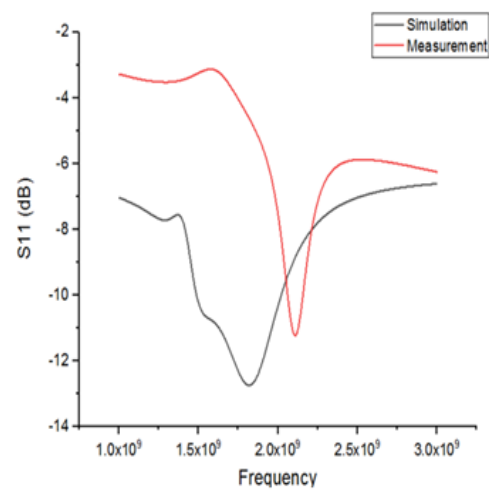

(b)

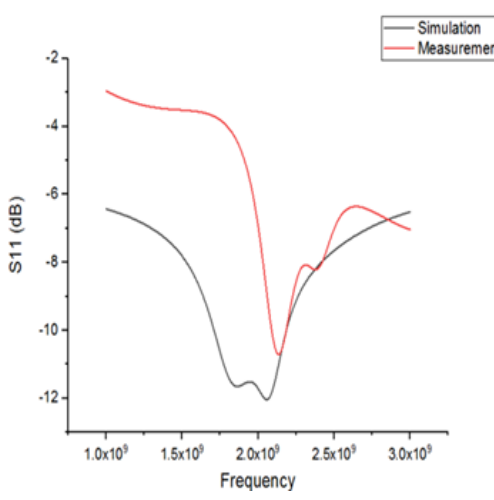

(c)

Figure 9. Comparison of simulated and measurement graph, (a) width $0.5 \mathrm{~mm}$, (b) width $0.6 \mathrm{~mm}$, (c) width $0.7 \mathrm{~mm}$

A comparison table of the resonance frequencies and the $Q$ factors for all three devices is shown in Table 2. The table also compares simulation and experimental results. It can be seen that while there is a frequency shift of the resonance frequencies between the simulated and measured results, the $Q$ factors for simulation and measurements are close to each other.

Table 2. Comparison table of resonance frequency and Q-factor for each design

\begin{tabular}{ccccccc}
\hline & \multicolumn{3}{c}{ Simulation } & \multicolumn{3}{c}{ Experimental } \\
\hline$w$ & $0.5 \mathrm{~mm}$ & $0.6 \mathrm{~mm}$ & $0.7 \mathrm{~mm}$ & $0.5 \mathrm{~mm}$ & $0.6 \mathrm{~mm}$ & $0.7 \mathrm{~mm}$ \\
$\begin{array}{c}\text { Frequency } \\
\text { (GHz) }\end{array}$ & 1.79 & 1.88 & 2.06 & 2.02 & 2.11 & 2.14 \\
Q-Factor & 10.02 & 10.13 & 10.41 & 10.63 & 11.72 & 5.156 \\
\hline
\end{tabular}

Modeling and development of radio frequency planar interdigital... (Muhammad Farhan Affendi bin Yunos) 


\section{DISCUSSION AND CONCLUSION}

In this paper, designs of interdigital sensors were simulated and measured. The interdigital sensor was first designed using theoretical equations and then simulated with CST Microwave Studio®. The widths of the interdigital sensors were varied to study which design produced the best response. The electric field simulations and S11 reflection characteristics were done to evaluate the best design. This sensor intends to be used for non-invasive sensing of blood glucose concentrations. Thus, its operating characteristics were set to be between 1-3 GHz. Initial simulations show that the resonance frequencies and its $\mathrm{Q}$ factors are satisfactory for this application. Next, the interdigital sensors were fabricated of the sensors on a FR4 board.

Experimental measurements were made using a vector network analyzer to validate the simulation results. The result was compared between simulation and measurement and it has shown slight difference in resonance frequencies. Larger widths will result in lower resonance frequencies. Further evaluations are required to improve the Q-factors. Higher Qs are important to produce sensors with higher sensitivities.

\section{ACKNOWLEDGEMENTS}

This work is supported by Malaysia Ministry of Higher Education (MOHE) under the Fundamental Research Grant Scheme (FRGS) FRGS17-030-0596

\section{REFERENCES}

[1] A. V. Mamishev, K. Sundara-Rajan, Fumin Yang, Yanqing Du and M. Zahn, "Interdigital sensors and transducers," in Proceedings of the IEEE, vol. 92, no. 5, pp. 808-845, May 2004

[2] A. A. Md Ralib and A. N. "Nordin. Silicon compatible acoustic wave resonators: Design, fabrication and performance". 2014, IIUM Engineering Journal, vol. 15, (2).

[3] J.W. Grate, G.C. Frye, in: H. Baltes, W. Göpel, J. Hesse (Eds.), Sensors, Update vol. 2, Wiley, New York, 1996, pp. 52-59.

[4] C. S. Operation, "Capacitive Sensor Operation and Optimization," no. November, 2015.

[5] M. Kitsara, D. Goustouridis, S. Chatzandroulis, M. Chatzichristidi, I. Raptis, Th. Ganetsos, R. Igreja, C.J. Dias. "Single chip interdigitated electrode capacitive chemical sensor arrays". Sens. Actuators, B 127 (1) (2007) 186-192.

[6] A.R. Mohd Syaifudin, S.C. Mukhopadhyay, P.L. Yu. "Novel sensors for food inspections". Sens. Trans. J. 114 (3) (2010) $1-40$

[7] S. Lei, C. Deng, Y. Chen, Y. Li. "A novel serial high frequency surface acoustic wave humidity sensor". Sens. Actuators, A 167 (2) (2011) 231-236.

[8] K. Chetpattananondh, T. Tapoanoi, P. Phukpattaranont, and N. Jindapetch. "A self-calibration water level measurement using an interdigital capacitive sensor". Sens. Actuators Phys., vol. 209, pp. 175-182, 2014.

[9] Zulkarnain Zulkarnain, Suprapto Suprapto, Taslim Ersam, Fredy Kurniawan. "A Novel Selective and Sensitive Electrochemical Sensor for Insulin Detection". Indonesian Journal of Electrical Engineering and Computer Science, 3(3), pp. 496-502.

[10] A. A. Zainuddin et al. "Verification of Quartz Crystal Microbalance Array using Vector Network Analyzer and OpenQCM". Indones. J. Electr. Eng. Comput. Sci., vol. 10, no. 1, pp. 84-93, 2018.

[11] E. Topsakal, T. Karacolak, and E. C. Moreland. "Glucose-dependent dielectric properties of blood plasma". 2011 URSI Gen. Assem. Sci. Symp., pp. 1-4, 2011.

[12] M. F. A. Yunos, A. N. Nordin, Sheroz Khan. "Simulation of RF Patch Antenna Sensor for Biological Applications". 2018 7th International Conference on Computer and Communication Engineering (ICCCE), Kuala Lumpur, 2018, pp. 62-67.

[13] Rahman, Mohd Syaifudin Abdul, Subhas Chandra Mukhopadhyay, and Pak-Lam Yu. "Novel planar interdigital sensors". Novel Sensors for Food Inspection: Modelling, Fabrication and Experimentation. Springer, Cham, 2014. 11-35.

[14] C. Méndez Ruiz et al., "Improve signal integrity performance by using hybrid PCB stackup," 2013 IEEE International Symposium on Electromagnetic Compatibility, Denver, CO, 2013, pp. 317-321.

[15] B. A. Cetiner, J. Y. Qian, H. P. Chang, M. Bachman, G. P. Li and F. De Flaviis, "Monolithic integration of RF MEMS switches with a diversity antenna on PCB substrate," in IEEE Transactions on Microwave Theory and Techniques, vol. 51, no. 1, pp. 332-335, Jan. 2003. 


\section{BIOGRAPHIES OF AUTHORS}
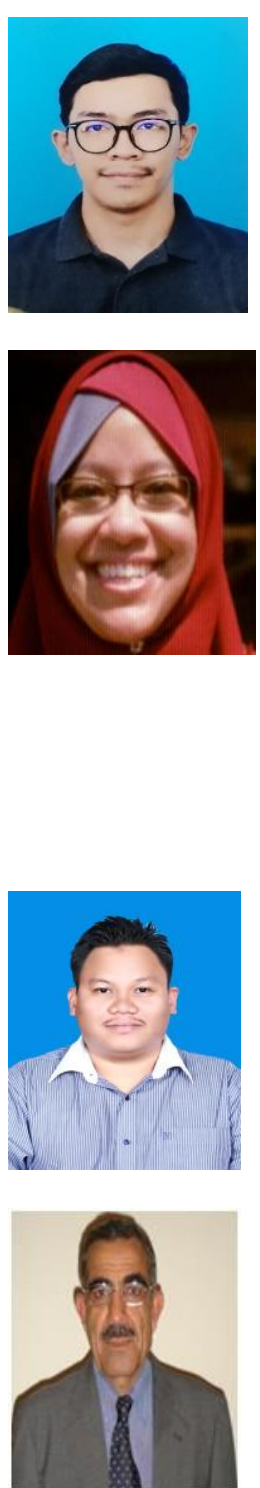

Muhammad Farhan Affendi received his bachelor's degree from International Islamic University Malaysia, (IIUM) in 2017. Currently, he is a master's degree candidate at Department of Electronic and Computer Engineering, International Islamic University Malaysia. His research interest are in microfluidics and modelling of MEMS devices.

Anis Nurashikin Nordin has a bachelor's degree from International Islamic University Malaysia (IIUM) in 2000. She obtained both her Master of Science and Doctor of Science in Computer Engineering from the George Washington University, Washington D.C. in 2002 and 2008 respectively. Her field of specialization, both in her Masters and doctorate degree is in Microelectronics and VLSI. Currently, she is an Associate Professor in the Department of Electrical and Computer Engineering at the International Islamic University Malaysia, Kuala Lumpur. She is passionate about her research and her main research interests are in the area of biosensors, microfluidics, lab-on-chip devices, Analog VLSI, RF-MEMS, and surface acoustic wave resonators. She has won several awards at the university level most recently Best Journal Article Award and Best Overall Researcher Award in 2018. She has also won numerous medals both internationally and locally for her research in developing personalized sensors for cancer chemotherapeutics. Among her awards from the International European Women Inventors and Innovators Network Awards, Malaysian Technological Expo, British Invention Show, EU Special Inventor Award in the British Invention Show, International Trade Fair, Ideas, Inventions, New Products, Germany.

Ahmad Anwar Zainuddin is a PhD candidate in International Islamic University Malaysia, (IIUM). $\mathrm{He}$ received his Bachelor's and Master's degree from Department of Electronic and Computer Engineering, International Islamic University Malaysia in 2011 and 2013, respectively. He worked as a MEMS engineer in Silterra Malaysia in 2011, before he becomes a PhD candidate in IIUM in 2015. His research interests include acoustic wave electrochemical biosensors, computational mechanics, structural dynamics, smart materials and structures, and modelling of MEMS devices.

Sheroz Khan received his B Sc. in Electrical Engineering from the N-W.F.P University of Engineering and Technology (NWF UET) Peshawar, Pakistan. Being the best graduate of the Department of Electrical Engineering in 1982, he was awarded the university scholarship (1988) for doing M Sc in Microelectronic \& Computer Engineering at Surrey University, UK. His timely accomplishment of masters (1990) at Surrey was acknowledged by the NWFP UET Senate Decision in the form of nominating him under the British Council program for further study at Strathclyde University, UK, which he completed his PhD to re-join his parent university in August 1994. Since 2000, he has been working within the department of ECE at the IIUM Kuala Lumpur. 